\title{
Achieving Wideband sub-1dB Noise Figure and High Gain with MOSFETs if Input Power Matching is not Required
}

\author{
Eric A.M. Klumperink ${ }^{1}$, Qiaohui Zhang ${ }^{1}$, Gerard J. M. Wienk ${ }^{1}$, Roel Witvers ${ }^{2}$, Jan Geralt Bij de Vaate $^{2}$, \\ Bert Woestenburg ${ }^{2}$, Bram Nauta ${ }^{1}$ \\ ${ }^{1}$ University of Twente, CTIT Research Institute, IC-Design Laboratory, Enschede, The Netherlands \\ ${ }^{2}$ Netherlands Foundation for Research in Astronomy (ASTRON), Dwingeloo, The Netherlands
}

\begin{abstract}
A 0.18 $\mu$ m CMOS Low Noise Amplifier (LNA) achieves sub-1dB Noise Figure over more than an octave of bandwidth without external noise matching components. It is designed for a future radio telescope, requiring millions of cheap LNAs mounted directly on phased array antenna elements. The short distance between antenna and LNA and low frequency below $2 \mathrm{GHz}$ allows for using an LNA with reflective input impedance, increasing the gain with $6 \mathrm{~dB}$. Without any matching network, very low noise figure is achieved over a wide bandwidth. At $90 \mathrm{~mW}$ power, sub-1dB Noise is achieved for $50 \Omega$ source impedance over a $0.8-1.8 \mathrm{GHz}$ band without external coils, and $S 21>20 \mathrm{~dB}$, OIP2 $>25 \mathrm{dBm}$ and $O I P 3>15 \mathrm{dBm}$. Preliminary results with $150 \Omega$ source impedance show noise temperatures as low as $25 \mathrm{~K}$ around $900 \mathrm{MHz}$.
\end{abstract}

Index Terms - Low Noise Amplifier, CMOS, Wideband Amplifier, Phased Array, radio receiver, radio astronomy.

\section{INTRODUCTION}

Deep submicron MOSFETs can achieve a minimum noise figure $\mathrm{NF}_{\text {min }}$ well below $0.5 \mathrm{~dB}$ for low $\mathrm{GHz}$ frequencies [1], equivalent to a noise temperature below $35 \mathrm{~K}$. Thus MOSFETs are becoming a viable alternative for ultra low-noise applications and are even considered for future radio astronomy applications, traditionally the domain for cryogenic cooled LNAs. This paper reports simulated and measured results of a CMOS LNA designed to push the limits, aiming at the lowest possible noise over more than an octave bandwidth for a $100 \mathrm{~mW}$ power budget. The LNA is designed for a "Square Kilometer Array" (SKA), a phased array antenna system with millions of antenna elements to achieve 100 times more sensitivity than the best telescope today. To achieve this sensitivity, high gain amplifiers with very low noise are attached directly to antenna elements, which means that millions of LNAs are needed. To realize such a system at reasonable costs, the use of low-cost mainstream technology is planned with antennas realized as printed circuits and CMOS LNAs mounted on them, preferably without any further external components. These boundary conditions make this paper also relevant for other low-cost wideband phased array applications.

In this paper we focus on a SKA-LNA for the $0.6-1.6 \mathrm{GHz}$ band. To achieve the desired sensitivity, the LNAs should have a $\mathrm{NF}<0.6 \mathrm{~dB}$ in combination with a gain of at least $20 \mathrm{~dB}$ to suppress the noise contributions of later stages sufficiently. Given the relatively low frequency, the LNA will be close enough to the antenna to neglect standing wave phenomena. Thus there seems to be no strict reason to require input impedance matching of the LNA to the antenna impedance, and a high ohmic highly reflective input impedance could be acceptable. However, output impedance matching is needed, as the LNA needs to drive a long $50 \Omega$ transmission line to a beam former, which will phase shift and combine multiple antenna signals of the phased array. In the wide 600$1600 \mathrm{MHz}$ band several strong interferers can occur, so both good 2nd and 3rd order linearity is desired.

This paper will discuss the design and experimental verification of a CMOS LNA aiming at wideband ultra low noise. Section II discusses LNA design considerations, motivating the choice for a reflective input impedance and high antenna impedance. Section III presents a $0.18 \mu \mathrm{m}$ CMOS circuit design. Experimental results are reported in section IV, while conclusions are drawn in section $\mathrm{V}$.

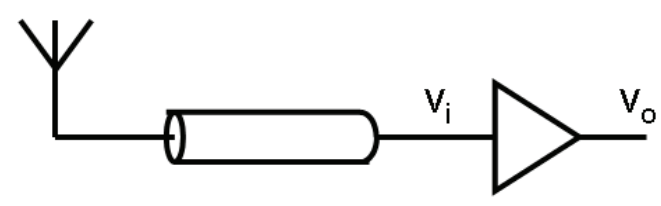

$$
\text { Model for short lines }
$$

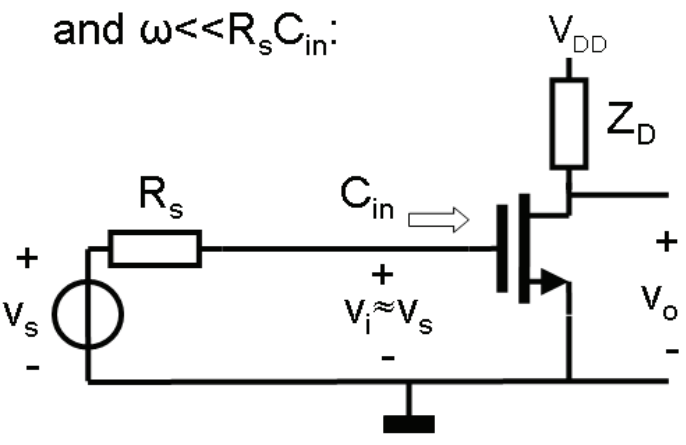

Fig. 1. Highly simplified Antenna with MOSFET LNA working in the low $\mathrm{GHz}$ range, mounted directly on an antenna element 


\section{LNA DESIGN CONSIDERATIONS}

Assuming the LNA is mounted close enough to the antenna elements and we don't require input impedance matching, we can exploit a common-source MOSFET with large transconductance and high drain impedance $Z_{D}$ (see figure 1) to simultaneously obtain very large gain and very low noise.

Assuming $\omega<<1 /\left(R_{s} C_{i n}\right)$, the full source voltage $v_{s}$ will be available at the gate, instead of $\mathrm{v}_{\mathrm{s}} / 2$ in case of impedance matching. This renders $6 \mathrm{~dB}$ more gain, reducing the noise contribution of later stages. Moreover, the usual trade-off between impedance matching and noise matching is avoided, allowing to push the noise figure to very low values. Technology extrapolations predict a minimum $\mathrm{NF}_{\text {min }}$ decreasing with Moore's law [1]: at $2 \mathrm{GHz}$ and a current density of $100 \mu \mathrm{A} / \mu \mathrm{m}$ gate-width, $\mathrm{NF}_{\text {min }}$ drops from roughly $0.4 \mathrm{~dB}$ for a $0.25 \mu \mathrm{m}$ technology to $0.1 \mathrm{~dB}$ for a $50 \mathrm{~nm}$ technology. However, note that this minimum is only achieved at an optimum source impedance with a large resistive and inductive component.

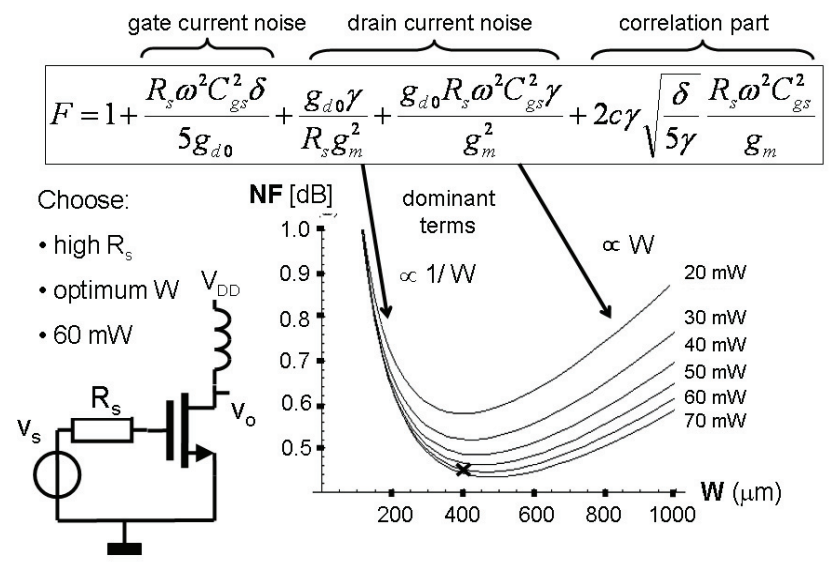

Fig. 2. Choices to optimize the noise factor $F$ of a single Common Source MOSFET and Noise Optimization for $\mathrm{R}_{\mathrm{s}}=150 \Omega$ (symbols as commonly used [2]).

For a real source impedance of $50 \Omega$, typical NF50 values are not much lower than $1 \mathrm{~dB}$ for a $0.18 \mu \mathrm{m}$ CMOS and $0.5 \mathrm{~dB}$ for a $50 \mathrm{~nm}$ technology [1]. Thus even $50 \mathrm{~nm}$ technology seems not good enough to achieve $<0.6 \mathrm{~dB}$ noise figure, as $0.1-0.2 \mathrm{~dB}$ noise budget is needed for other sources of noise, like the noise contribution of the load impedance, the $50-\Omega$ driver stage and unavoidable losses in the connections. Unfortunately, the use of a simple gate series-inductor is not of great help. Citing [2] $\mathrm{B}_{\mathrm{op}} \approx-\omega \mathrm{C}_{\mathrm{gs}}$ : "We see that the optimum source susceptance is essentially inductive in character, except that it has the wrong frequency behavior. Hence, achieving a broadband noise match is fundamentally difficult." Indeed, all fully integrated CMOS LNAs with sub-1dB NF that we found in literature achieve low noise in a bandwidth which is only a small fraction of the center frequency.
Another problem associated with inductive noise matching networks is the need for large inductors, to realize the optimum source impedance for a very wide high transconductance MOSFET. To be effective at $600 \mathrm{MHz}$, inductors in the order of $10 \mathrm{nH}$ or more are needed. As $1 \Omega$ series resistance already renders $0.09 \mathrm{~dB} \mathrm{NF}$ contribution for $50 \Omega$, inductor Q-values of about 100 are needed, far from feasible on a CMOS chip. However, one option is still left: according to the noise factor equation in figure 2 , raising the source resistance can be quite effective. Experiments with several antenna designs show that a real impedance level in the order of $150 \Omega$ is feasible, and we optimized our LNA for that antenna impedance. The transistor width and bias was optimized at various frequencies using a power constraint noise optimization similar to [2]. Figure 2 shows a typical result for a $0.18 \mu \mathrm{m}$ MOSFET and indicates the dominant terms. A rather flat optimum width of about $400 \mu \mathrm{m}$ was found. To push the noise limit, a bias of $60 \mathrm{~mW}$ at $1.8 \mathrm{~V}$ was chosen $(\sim 75 \mu \mathrm{A} / \mu \mathrm{m}$, close to the optimum predicted by [1]).

\section{CMOS LNA DESIGN}

In order to minimize noise and obtain more then $20 \mathrm{~dB}$ gain, cascoding and a combination of a drain resistor and inductance is used. The drain inductor results in a strong increase of the gain with frequency, which degrades linearity and is problematic in the further signal processing. To equalize the gain somewhat, inductive degeneration was used in the second stage (see figure 3).

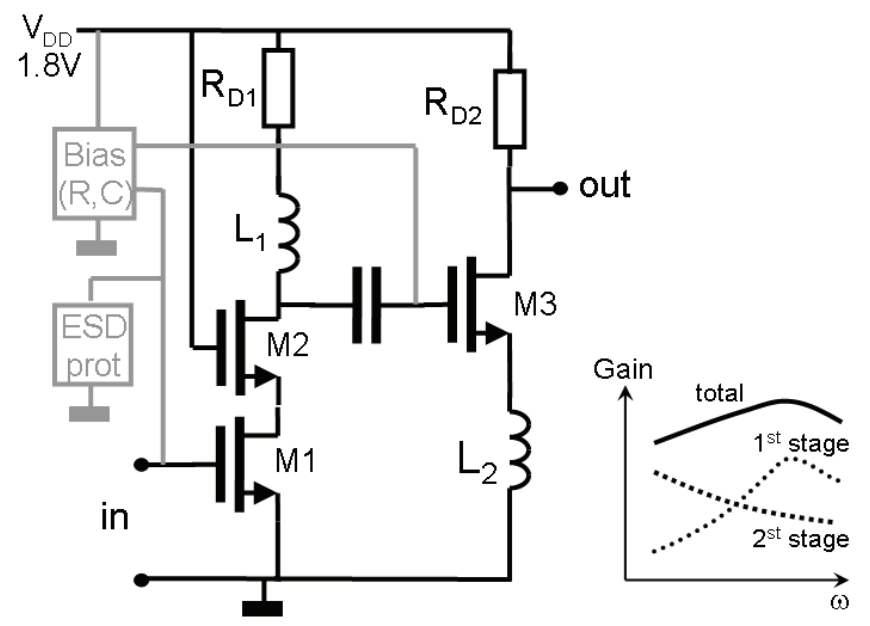

Fig. 3: Schematic of the LNA-IC with gain-variation reduction via the inductor $\mathrm{L}_{2}$

Figure 4 shows simulation results for the LNA with $50 \Omega$ and $150 \Omega$ source impedance using UMC18 RF models. For $150 \Omega$ source impedance, the noise figure remains below $0.6 \mathrm{~dB}$ from $300 \mathrm{MHz}$ to $1.5 \mathrm{GHz}$. For $50 \Omega$ the minimum noise is about $0.8 \mathrm{~dB} @ 1.5 \mathrm{GHz}$ with sub- $1 \mathrm{~dB}$ noise figure from 
$600 \mathrm{MHz}$ to above $2 \mathrm{GHz}$. Note that this includes noise of the RC-bias circuit. Almost all LNA noise originates from M1 (e.g. $>93 \%$ for $0.8 \mathrm{~dB} @ 1.5 \mathrm{GHz}$ ). The gain equalization reduces the gain variation over the $0.6-1.6 \mathrm{GHz}$ band to $<3 \mathrm{~dB}$ $(150 \Omega)$ and $<7 \mathrm{~dB}(50 \Omega)$.

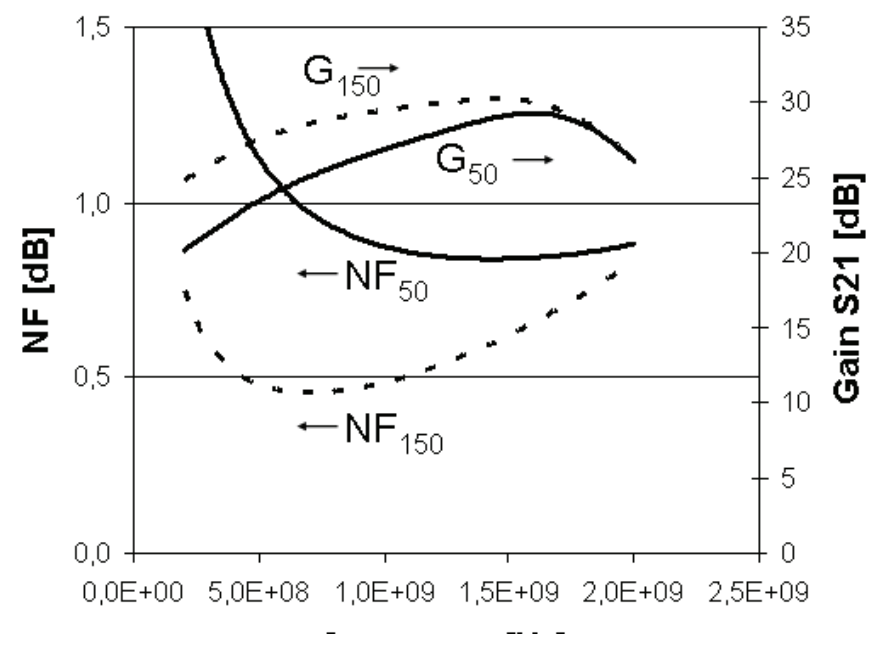

Fig. 4: Simulated Gain $\mathrm{S} 21$ and NF for $50 \Omega$ and $150 \Omega$ source impedance.

\section{EXPERIMENTAL RESULTS}

The chip was fabricated at UMC and its photograph is shown in figure 5. At this point in time, only well understood measurement results for a $50 \Omega$ source impedance are available, and we are still working on the $150 \Omega$ measurements. Figure 6 shows the s-parameters measured via wafer-probing.

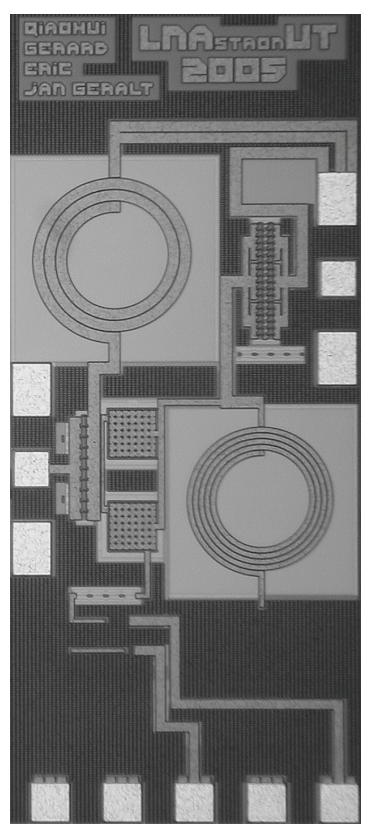

Fig. 5: Chip photo of the wideband LNA $(0.60 \times 1.2 \mathrm{~mm} 2)$
There are some deviations w.r.t. simulation, rendering somewhat more peaking in the gain and worse S22 due to a (too) high RD2 value. The noise figure was measured in many ways with several double-checks, both on-wafer and on a board with the option to add external inductors. The best reproducible results were obtained in a Faraday Room for board measurements using a Agilent N8973A with a Agilent 346A noise source. Figure 7 shows that measurement results without external inductor resemble simulations (figure 4), except for a deviation at high frequencies which is most probably caused by the bond-wire inductance. To verify the measurement setup and the theoretical expectations, measurements with high-Q external series inductors were also done $(4 \mathrm{nH}$ and $10 \mathrm{nH})$. As expected, increasing the gate inductance helps to decrease the minimum noise figure (from $0.8 \mathrm{~dB}$ to $0.6 \mathrm{~dB}$ ), but also limits the low-noise bandwidth. The best bandwidth is achieved without external inductors, with more then $1 \mathrm{GHz}$ sub-1 $\mathrm{dB}$ noise figure bandwidth. Table I compares both simulations and measurements to other designs, showing the competitiveness of this design in lownoise bandwidth.

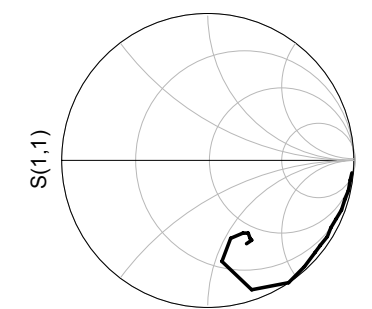

freq $(100.0 \mathrm{MHz}$ to $2.000 \mathrm{GHz})$

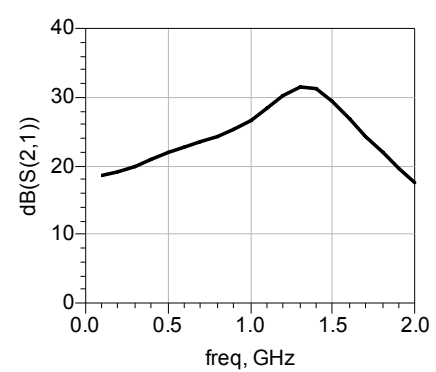

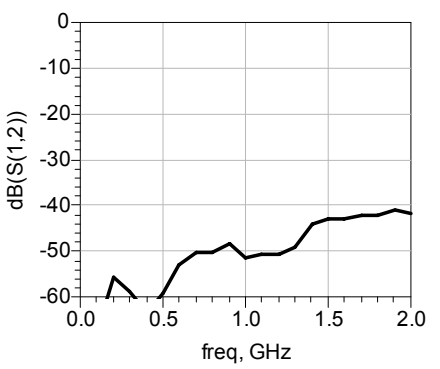

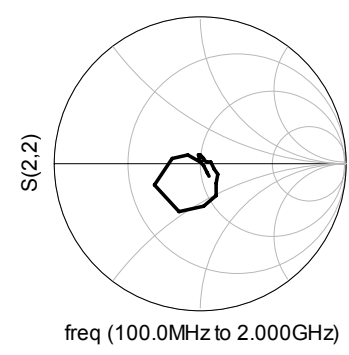

Fig. 6: Measured S-parameters of the LNA with respect to $50 \Omega$

Noise measurements for variable source impedance are currently ongoing, where we use a $50 \Omega, 100 \Omega$ and $150 \Omega$ resistor which is cooled down with liquid Nitrogen to $77 \mathrm{~K}$ to realize a "cold" measurement, while doing a "hot" measurement at room temperature. Measurements for this method agree well with the $50 \Omega$ results in figure 7 . For $100 \Omega$ and $150 \Omega$ source impedance, we measure lower noise figure, as expected. At $900 \mathrm{MHz}$ and $150 \Omega$ source impedance we reproducibly measure a noise temperature as low as $25 \mathrm{~K}$ $(0.35 \mathrm{~dB})$. However, above $1 \mathrm{GHz}$ the noise figure rises sharply. We currently try to resolve whether this is caused by non modeled parasitic components in the IC, or by the measurement setup (the current setup still requires an 
electrically long transmission line to connect to the $150 \Omega$ resistance in the dewar with liquid nitrogen, and we also use a $92 \Omega$ semi-rigid coaxial line for reasons of practical availability).

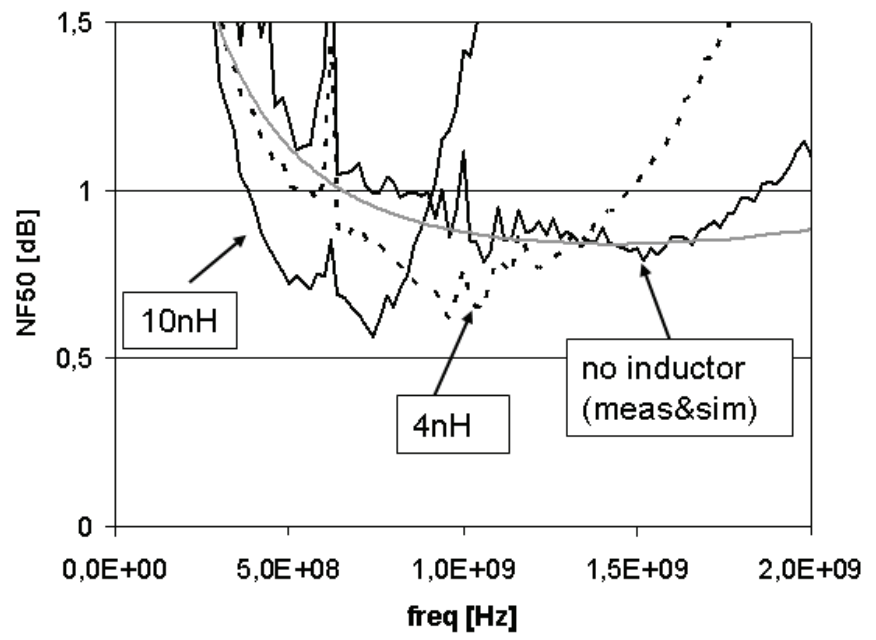

Fig. 7: NF50 measured using a bonded LNA on a board with and without high-Q external inductors

\section{CONCLUSIONS}

In this paper we report work that tries to push the noise figure and gain of a wideband 500MHz-1.6GHz CMOS LNA, assuming we don't need input impedance matching because the LNA is mounted an antenna with very short connection lines. We motivated why a Common Source MOSFET without any noise matching network is suitable to achieve wideband low noise without any external high-Q components, while a high antenna impedance is favorable to reduce the noise figure. Measurements show that even in a $0.18 \mu \mathrm{m}$ CMOS technology sub-1dB noise figure and more than $23 \mathrm{~dB}$ gain can be achieved over more than an octave in bandwidth $(0.8-1.8 \mathrm{GHz})$ at $90 \mathrm{~mW}$ power consumption. For $150 \Omega$ source impedance, a narrowband noise figure of $0.35 \mathrm{~dB}$ has been measured at $900 \mathrm{MHz}$, but further wideband noise characterization is needed.

Acknowledgement - The authors like to thank Henk de Vries for his support during the tedious noise measurements.

\section{REFERENCES}

[1] P. H. Woerlee et al, "RF-CMOS performance trends," IEEE Tr. on Electron Devices, pp. 1776-1782, 2001.

[2] T. H. Lee, "The Design of CMOS Radio-Frequency Integrated Circuits", Cambridge Univ. Press, 2003

[3] J. $\mathrm{Xu}$ et al, "GaAs $0.5 \mathrm{~dB}$ NF dual-loop negative-feedback broadband low-noise amplifier IC," Electronics Letters, pp. 780$782,2005$.

[4] L. Belostotski et al, "Wide-band CMOS Low Noise Amplifier for Applications in Radio Astronomy," ISCAS, pp. 1347-1350, 2006.

[5] F. Bruccoleri et al, "Wide-Band CMOS Low-Noise Amplifier Exploiting Thermal Noise Canceling," JSSC, pp. 275-281, 2004.

[6] J.-H. C. Zhan and S. S. Taylor, "A 5 GHz Resistive-Feedback CMOS LNA for Low-Cost Multi-Standard Applications," ISSCC, pp. 200-201, 2006.

[7] G. Gramegna et al, "A sub-1-dB NF\&plusmn;2.3-kV ESDprotected 900-MHz CMOS LNA," JSSC,, pp. 1010-1017, 2001.

[8] P. Leroux et al, "A 0.8-dB NF ESD-Protected 9-mW CMOS LNA operating at $1.23 \mathrm{GHz}$ [for GPS receiver]," JSSC, pp. 760-765, 2002.

TABLE I

PERFORMANCE COMPARISON WITH OTHER LOW NOISE AMPLIFIER DESIGNS.

\begin{tabular}{|c|c|c|c|c|c|c|c|c|}
\hline Property & $\begin{array}{c}\text { This } \\
150 \Omega\end{array}$ & [3] & [4] & $\begin{array}{l}\text { This } \\
50 \Omega \\
\end{array}$ & [5] & [6] & [7] & {$[8]$} \\
\hline Gain [dB] & $>26$ & $>21$ & $>18$ & $>23$ & 14 & 25 & 14 & 20 \\
\hline lowest NF [dB] & 0.35 meas & 0.45 & 0.45 & 0.8 & 1.8 & 1.9 & 0.85 & 0.8 \\
\hline $\begin{array}{l}\text { low noise band } \\
{[\mathrm{GHz}] \text { for NF: }}\end{array}$ & $\begin{array}{c}0.3 \ldots 1.4 \\
<.6 \mathrm{~dB}\end{array}$ & $\begin{array}{c}0.6 \ldots 1.6 \\
<.5 \mathrm{~dB}\end{array}$ & $\begin{array}{c}0.7 \ldots 1.5 \\
<.6 \mathrm{~dB}\end{array}$ & $\begin{array}{c}0.8 \ldots 1.8 \\
<1 \mathrm{~dB}\end{array}$ & $\begin{array}{c}0.2 \ldots 1.1 \\
<2 \mathrm{~dB}\end{array}$ & $\begin{array}{c}1.0 \ldots 4.5 \\
<2 \mathrm{~dB}\end{array}$ & $\begin{array}{c}0.85 \ldots 0.95 \\
\quad<1 \mathrm{~dB}\end{array}$ & $\begin{array}{c}1.1 \ldots 1.3 \\
<1 \mathrm{~dB}\end{array}$ \\
\hline OIP2 [dBm] & $\begin{array}{c}28 \\
1.8 \& .8 \mathrm{G}\end{array}$ & $?$ & $?$ & $\begin{array}{c}25 \\
1.8 \& .8 \mathrm{G}\end{array}$ & 26 & - & - & - \\
\hline OIP3 [dBm] & $\begin{array}{c}14 \\
1.1 \& 1.2 \mathrm{G}\end{array}$ & 15 & $?$ & $\begin{array}{c}15 \\
1.1 \& 1.2 \mathrm{G}\end{array}$ & 14 & 21 & 16 & +9 \\
\hline Power [mW] & 90 & 850 & 50 & 90 & 35 & 42 & 18 & 9 \\
\hline Area $\left[\mathrm{mm}^{2}\right]$ & 0.72 & 1.5 & $?$ & 0.72 & 0.075 & $\sim .025$ & .66 & .66 \\
\hline External coils? & 0 & 1 & 3 & $\begin{array}{c}0 \\
\text { bondwire }\end{array}$ & 0 & 0 & 1 & $\begin{array}{c}0 \\
\text { bondwire } \\
\end{array}$ \\
\hline $\begin{array}{l}\text { CMOS } \\
\text { Process }\end{array}$ & $.18 \mu$ & $\begin{array}{c}.2 \mu \\
\mathrm{GaAs}\end{array}$ & $.18 \mu$ & $.18 \mu$ & $.25 \mu$ & $.09 \mu$ & $.35 \mu$ & $.25 \mu$ \\
\hline meas/sim & $\operatorname{sim}$ & $\operatorname{sim}$ & $\operatorname{sim}$ & meas & meas & meas & meas & meas \\
\hline
\end{tabular}

